02

\title{
Локальная структура и процессы гидратации галогензамещенных перовскитов на основе $\mathrm{Ba}_{4} \ln _{2} \mathrm{Zr}_{2} \mathrm{O}_{11}$
}

\author{
(C) Н.А. Тарасова, А.О. Галишева, И.Е. Анимица \\ Уральский федеральный университет им. первого Президента России Б.Н. Ельцина, \\ Институт естественных наук и математики, \\ 620002 Екатеринбург, Россия \\ e-mail: Natalia.Tarasova@urfu.ru
}

Поступила в Редакцию 03.09.2018 г.

В окончательной редакции 03.09.2018 г.

Принята к публикации 11.12.2018 г.

Осуществлен синтез галогензамещенных перовскитов $\mathrm{Ba}_{4} \mathrm{In}_{2} \mathrm{Zr}_{2} \mathrm{O}_{10.95} \mathrm{~F}_{0.1}$ и $\mathrm{Ba}_{4} \mathrm{In}_{2} \mathrm{Zr}_{2} \mathrm{O}_{10.95} \mathrm{Cl}_{0.1}$, рентгенографически подтверждена их однофазность. Доказана способность исследуемых фаз к гидратации и формированию в структуре энергетически неравноценных $\mathrm{OH}_{-}$-групп. Установлено, что введение галогенидионов приводит к уменьшению степени гидратации относительно матричного состава, что объяснено уменьшением свободного объема кристаллической ячейки.

DOI: $10.21883 /$ OS.2019.04.47509.266-18

\section{Введение}

Метод введения галогенид-ионов в кислородную подрешетку сложного оксида с целью изменения структуры и улучшения физико-химических свойств широко используется для различных классов перовскитоподобных систем, таких, например, как сегнетоэлектрические и пьезоэлектрические материалы [1-4], сверхпроводники [5-8], магнитные материалы $[9,10]$, катодные материалы [11-13].

На протяжении последних лет метод анионного гетеровалентого допирования стал успешно применяться для оптимизации транспортных свойств кислородноионных и протонных проводников с перовскитоподобной структурой. Так, было установлено, что введение галогенид-ионов в анионную подрешетку кислород-дефицитных сложных оксидов позволяет повысить кислородноионную и протонную проводимости, а также улучшить химическую стабильность к углекислому газу [14-16].

Как известно, наличие вакансий кислорода в соединениях со структурой перовскита $\mathrm{ABO}_{3-\delta}$ или производной от нее обеспечивает возможность транспорта ионов кислорода в сухой атмосфере и протонов во влажной. Максимальный кислородный дефицит реализуется у соединений состава $\mathrm{A}_{2} \mathrm{~B}_{2} \mathrm{O}_{5} \equiv \mathrm{ABO}_{2,5}$. Хотя для них большие концентрации вакансий кислорода обеспечивают значимые концентрации протонов, высокие значения кислород-ионной и протонной проводимостей не реализуются из-за упорядочения дефектов. Однако между составами $\mathrm{ABO}_{3}$ и $\mathrm{A}_{2} \mathrm{~B}_{2} \mathrm{O}_{5}$ возможно формирование индивидуальных фаз промежуточного состава, например $\mathrm{A}_{4} \mathrm{~B}_{2}{ }_{2} \mathrm{~B}_{2}{ }_{2} \mathrm{O}_{11}$, в которых 1/12 кислородных позиций вакантна. Для этих концентраций дефектов реализуется их статистическое расположение и, как следствие, высокие подвижности, поэтому фазы такого состава представляют интерес для исследования.
В настоящей работе были получены фтор- и хлорзамещенные составы на основе кислород-дефицитного состава $\mathrm{Ba}_{4} \mathrm{In}_{2} \mathrm{Zr}_{2} \mathrm{O}_{11}$, ранее описанного как высокотемпературный протонный проводник [17]. Исследовано влияние природы галогенид-ионов на локальную структуру и процессы гидратации.

\section{Экспериментальная часть}

Образцы $\mathrm{Ba}_{4} \mathrm{In}_{2} \mathrm{Zr}_{2} \mathrm{O}_{10.95} \mathrm{~F}_{0.1} \quad$ и $\mathrm{Ba}_{4} \mathrm{In}_{2} \mathrm{Zr}_{2} \mathrm{O}_{10.95} \mathrm{Cl}_{0.1}$ были получены методом твердофазного синтеза из $\mathrm{BaCO}_{3}, \mathrm{In}_{2} \mathrm{O}_{3}, \mathrm{ZrO}_{2}, \mathrm{BaF}_{2}$ и $\mathrm{BaCl}_{2}$. Синтез проводили на воздухе при ступенчатом повышении температуры $\left(800-1300^{\circ} \mathrm{C}\right)$ и многократных перетираниях в агатовой ступке в среде этилового спирта. Время отжига на каждой стадии составляло $24 \mathrm{~h}$. Состав $\mathrm{Ba}_{4} \mathrm{In}_{2} \mathrm{Zr}_{2} \mathrm{O}_{11}$ синтезирован для сравнения по аналогичной схеме.

Рентгенографический анализ был выполнен на дифрактометре Bruker Advance D8 в CuK-излучении при напряжении на трубке $40 \mathrm{kV}$ и токе $40 \mathrm{~mA}$. Съемка производилась в интервале $2 \theta=20^{\circ}-80^{\circ}$ с шагом по $\theta$ $0.05^{\circ}$ и экспозицией $1 \mathrm{~s}$ на точку. Расчеты параметров решетки проводили с помощью программы FullProf Suite.

Исследование локальной структуры образцов было проведено методом спектроскопии комбинационного рассеяния (KP) на спектрометре WiTec Alpha 300 AR (синий лазер, $\lambda=488 \mathrm{~nm}$ ), 10-кратное увеличение, выдержка $5 \mathrm{~s}$, усреднение трех спектров.

Термический анализ проводили на приборе NETZSCH STA 409 РC в интервале температур $25-1000^{\circ} \mathrm{C}$ и скоростью нагрева $10^{\circ} \mathrm{C} / \mathrm{min}$. Перед измерениями образцы подвергались термической обработке во влажной атмосфере $\left(p_{\mathrm{H}_{2} \mathrm{O}}=2 \cdot 10^{-2} \mathrm{~atm}\right)$ путем медленного охлаждения от 1000 до $200^{\circ} \mathrm{C}$ со скоростью $1^{\circ} \mathrm{C} / \mathrm{min}$ с целью получения гидратированных форм образцов. 
Влажность задавали путем барботирования воздуха при комнатной температуре последовательно через дистиллированную воду и насыщенный раствор бромида калия $\operatorname{KBr}\left(p_{\mathrm{H}_{2} \mathrm{O}}=2 \cdot 10^{-2} \mathrm{~atm}\right)$.

Для идентификации кислородно-водородных групп использовали метод ИК спектроскопии. Исследования проводили на ИК фурье-спектрометре Nicolet 6700 в диапазоне частот от 400 до $4000 \mathrm{~cm}^{-1}$ методом диффузного отражения с использованием приставки Smart Diffuse Reflectance.

\section{Результаты и обсуждение}

Методом рентгенофазового анализа было установлено, что составы $\mathrm{Ba}_{4} \mathrm{In}_{2} \mathrm{Zr}_{2} \mathrm{O}_{10.95} \mathrm{~F}_{0.1}$ и $\mathrm{Ba}_{4} \mathrm{In}_{2} \mathrm{Zr}_{2} \mathrm{O}_{10.95} \mathrm{Cl}_{0.1}$ являются однофазными, характеризуются структурой одинарного перовскита (пространственная группа $P(m 3 m)$ и изоструктурны матричному составу $\mathrm{Ba}_{4} \mathrm{In}_{2} \mathrm{Zr}_{2} \mathrm{O}_{11}$. В качестве примера полнопрофильной обработки полученных рентгенограмм на рис. 1 представлены результаты для образца $\mathrm{Ba}_{4} \mathrm{In}_{2} \mathrm{Zr}_{2} \mathrm{O}_{10.95} \mathrm{Cl}_{0.1}$. Параметры элементарной ячейки галогензамещенных образцов и недопированного состава приведены в таблице. Необходимо отметить, что значения для $\mathrm{Ba}_{4} \mathrm{In}_{2} \mathrm{Zr}_{2} \mathrm{O}_{11}$ хорошо соотносятся с ранее полученными данными [17]. Как видно, введение в кислородную подрешетку иона с меньшим радиусом $\left(\mathrm{F}^{-}\right)$приводит к уменьшению параметров и объема ячейки, а введение иона с большим радиусом $\left(\mathrm{Cl}^{-}\right)$- к увеличению параметров и объема $\left(r_{\mathrm{O}^{2-}}=1.40 \AA, r_{\mathrm{F}^{-}}=1.33 \AA, r_{\mathrm{Cl}^{-}}=1.81 \AA[18]\right)$.

Локальная структура галогензамещенных перовскитов была исследована с помощью спектроскопии КР. КР-спектры образцов, характеризующихся идеальной кубической структурой, не должны содержать сигналов [19]. Однако локальные структурные искажения кубической структуры, такие, например, как вакансии

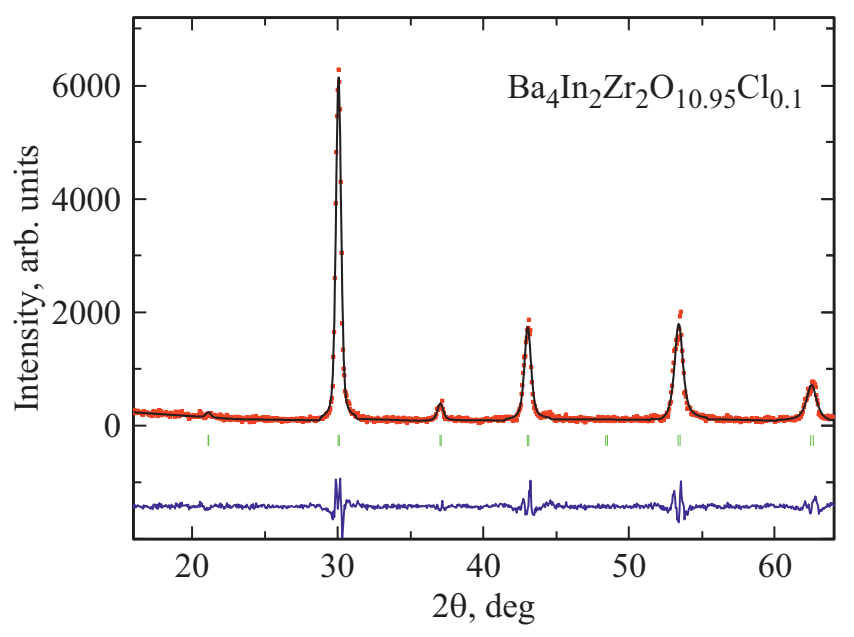

Рис. 1. Рентгенограмма образца $\mathrm{Ba}_{4} \mathrm{In}_{2} \mathrm{Zr}_{2} \mathrm{O}_{10.95} \mathrm{Cl}_{0.1}$. Показаны экспериментальные (точки), расчетные (линия), разностные (внизу) данные и угловые положения рефлексов (штрихи).

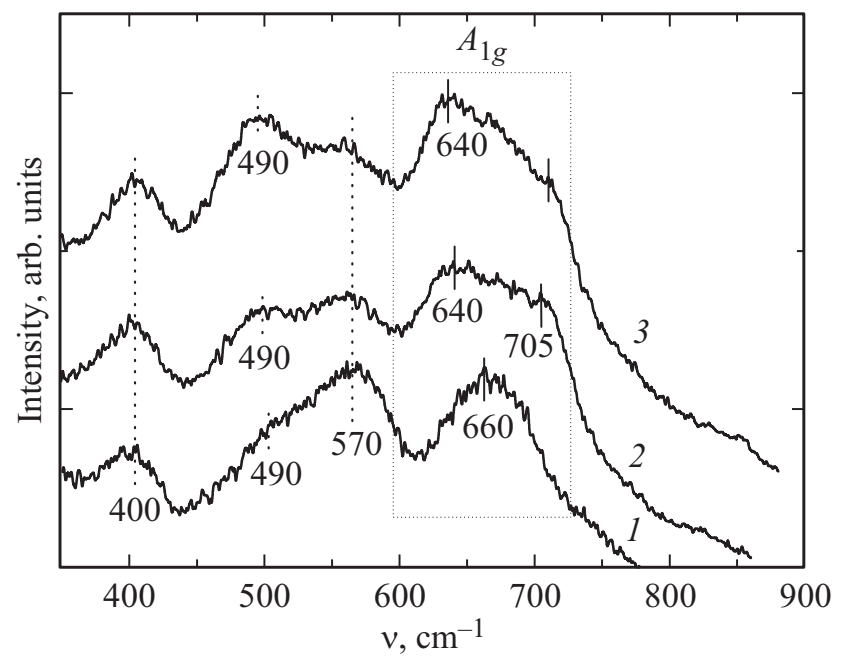

Рис. 2. КР-спектры безводных образцов $\mathrm{Ba}_{4} \mathrm{In}_{2} \mathrm{Zr}_{2} \mathrm{O}_{11}(1)$, $\mathrm{Ba}_{4} \mathrm{In}_{2} \mathrm{Zr}_{2} \mathrm{O}_{10.95} \mathrm{~F}_{0.1}$ (2) и $\mathrm{Ba}_{4} \mathrm{In}_{2} \mathrm{Zr}_{2} \mathrm{O}_{10.95} \mathrm{Cl}_{0.1}$ (3).

Параметры и объем элементарной ячейки исследуемых образцов

\begin{tabular}{c|c|c|c}
\hline Формула & $A, \AA$ & $V_{\text {sel }}, \AA^{3}$ & $V_{\text {free }}, \AA^{3}$ \\
\hline $\mathrm{Ba}_{4} \mathrm{In}_{2} \mathrm{Zr}_{2} \mathrm{O}_{11}$ & $4.204(3)$ & 74.30 & 23.37 \\
$\mathrm{Ba}_{4} \mathrm{In}_{2} \mathrm{Zr}_{2} \mathrm{O}_{10.95} \mathrm{~F}_{0.1}$ & $4.203(5)$ & 74.25 & 23.22 \\
$\mathrm{Ba}_{4} \mathrm{In}_{2} \mathrm{Zr}_{2} \mathrm{O}_{10.95} \mathrm{Cl}_{0.1}$ & $4.205(2)$ & 74.35 & 22.94
\end{tabular}

кислорода, приводят к появлению сигналов в спектрах исследуемых образцов.

На рис. 2 представлены КР-спектры безводных галогензамещенных перовскитов $\mathrm{Ba}_{4} \mathrm{In}_{2} \mathrm{Zr}_{2} \mathrm{O}_{10.95} \mathrm{~F}_{0.1}$ и $\mathrm{Ba}_{4} \mathrm{In}_{2} \mathrm{Zr}_{2} \mathrm{O}_{10.95} \mathrm{Cl}_{0.1}$ в сравнении со спектром недопированного состава $\mathrm{Ba}_{4} \mathrm{In}_{2} \mathrm{Zr}_{2} \mathrm{O}_{11}$. В спектрах исследуемых образцов можно выделить области, отвечающие валентным $\left(\sim 600-700 \mathrm{~cm}^{-1}\right)$ и деформационным $\left(\sim 400 \mathrm{~cm}^{-1}\right)$ колебаниям октаэдров [( $\left.\left.\mathrm{Zr} / \mathrm{In}\right) \mathrm{O}_{6}\right]$. Известно, что для перовскитоподобных структур полносимметричное валентное колебание $A_{1 g}$ всегда проявляется в виде сильного пика в области $600-800 \mathrm{~cm}^{-1}$. Частота колебания определяется природой катионов В' и В”, а также расстояниями В'-O и В”-О. С другой стороны, очевидно, что в исследуемых фазах из-за некомплектности кислородной подрешетки часть катионов в В-позициях будут иметь пониженные координационные числа, что приводит к появлению дополнительных пиков в высокочастотной области. Сравнение с КР-спектрами индата бария $\mathrm{Ba}_{2} \mathrm{In}_{2} \mathrm{O}_{5}$ [20], структура которого характеризуется чередованием тетраэдров $\left[\mathrm{InO}_{4}\right]$ и октаэдров $\left[\mathrm{InO}_{6}\right]$, позволяет сделать вывод о принадлежности пика $\sim 660 \mathrm{~cm}^{-1}$ (по форме и положению) валентным колебаниям тетраэдров $\left[\mathrm{InO}_{4}\right][21,22]$. В то же время колебательная мода для октаэдров $\left[\mathrm{ZrO}_{6}\right]$, соответствующая положению $745 \mathrm{~cm}^{-1}$ [23], вероятно, маскируется высокочастотной частью сигнала $\sim 660 \mathrm{~cm}^{-1}$. 
Как видно, введение галогенид-ионов не оказывает влияния на положение и форму сигнала, отвечающего деформационным колебаниям, в то время как линии валентных колебаний претерпевают значительные изменения. Как для фтор-, так и для хлорзамещенного образца происходит значительное расщепление полосы валентных колебаний $\mathrm{In}-\mathrm{O}$ на две составляющие: при этом основной максимум смещается в низкочастотную область $\sim 640 \mathrm{~cm}^{-1}$, а со стороны высоких частот появляется плечо $\sim 705 \mathrm{~cm}^{-1}$. Наряду с этим в спектрах галогензамещенных составов низкочастотное плечо сигнала $\sim 570 \mathrm{~cm}^{-1}$ становится более выраженным $\left(\sim 490 \mathrm{~cm}^{-1}\right)$. Это свидетельствует о значительном искажении полиэдров $\left[\mathrm{InO}_{4}\right]$ и появлении связей $\mathrm{In}-\mathrm{O}$ c разными длинами.

Возможность диссоциативного поглощения воды из газовой фазы для исследуемых образцов обусловлена наличием кислородных вакансий:

$$
\mathrm{H}_{2} \mathrm{O}+\mathrm{O}_{\mathrm{O}}^{\mathrm{x}}+\mathrm{V}_{\mathrm{O}}^{\mathrm{x}} \Leftrightarrow \mathrm{OH}_{\mathrm{O}}^{\bullet}+\mathrm{OH}_{\mathrm{V}_{\mathrm{O}}}^{\prime},
$$

где $\mathrm{O}_{\mathrm{O}}^{\mathrm{x}}-$ кислород в кристаллографической позиции кислорода, $\mathrm{V}_{\mathrm{O}}^{\mathrm{x}}$ - структурная вакансия кислорода, $\mathrm{OH}_{\mathrm{O}}^{\bullet}$ - гидроксо-группа, образующаяся в результате локализации протона на кислороде, стоящем в регулярной позиции, $\mathrm{OH}_{\mathrm{V}_{\mathrm{O}}}^{\prime}$ - гидроксо-группа, пришедшая от молекулы воды и занявшая структурную вакансию кислорода. Поэтому образцы подвергались термообработке во влажном воздухе для получения гидратированных форм.

Термогравиметрические исследования гидратированных образцов $\mathrm{Ba}_{4} \mathrm{In}_{2} \mathrm{Zr}_{2} \mathrm{O}_{11} \cdot n \mathrm{H}_{2} \mathrm{O}$, $\mathrm{Ba}_{4} \mathrm{In}_{2} \mathrm{Zr}_{2} \mathrm{O}_{10.95} \mathrm{~F}_{0.1} \cdot n \mathrm{H}_{2} \mathrm{O}$ и $\mathrm{Ba}_{4} \mathrm{In}_{2} \mathrm{Zr}_{2} \mathrm{O}_{10.95} \mathrm{Cl}_{0.1} \cdot n \mathrm{H}_{2} \mathrm{O}$ показали, что все составы способны к интеркаляции воды. Однако если для матричного состава предел гидратации, обусловленный концентрацией вакансий кислорода, достигается почти полностью и составляет $\sim 0.95 \mathrm{~mol} \mathrm{на} \mathrm{формульную} \mathrm{единицу} \mathrm{вещества,} \mathrm{то} \mathrm{для}$ фтор- и хлорзамещенных составов степени гидратации достигает $\sim 0.8$ и $\sim 0.6 \mathrm{~mol}$ соответственно. То есть введение галогенид-ионов приводит к уменьшению степени гидратации относительно недопированного состава. Для объяснения данного факта был рассчитан свободный объем элементарной ячейки исследуемых образцов (таблица). Он определяется как разность между общим объемом ячейки и суммой объемов, занимаемых индивидуальными ионами в ячейке и может быть рассчитан по следующей формуле:

$$
\underset{\text { элементарной ячейки }}{\text { свободный объем }}=a^{3}-\sum m_{i}(4 / 3) \pi r_{i}^{3} \text {, }
$$

где $a$ - это параметр решетки, $m_{i}-$ коэффициент химического состава, $r_{i}$ - ионный радиус.

B ряду $\mathrm{Ba}_{4} \mathrm{In}_{2} \mathrm{Zr}_{2} \mathrm{O}_{11}-\mathrm{Ba}_{4} \mathrm{In}_{2} \mathrm{Zr}_{2} \mathrm{O}_{10.95} \mathrm{~F}_{0.1}-$ $-\mathrm{Ba}_{4} \mathrm{In}_{2} \mathrm{Zr}_{2} \mathrm{O}_{10.95} \mathrm{Cl}_{0.1}$ происходит уменьшение свободного объема элементарной ячейки, что, очевидно, приводит к геометрическим затруднениям встраивания

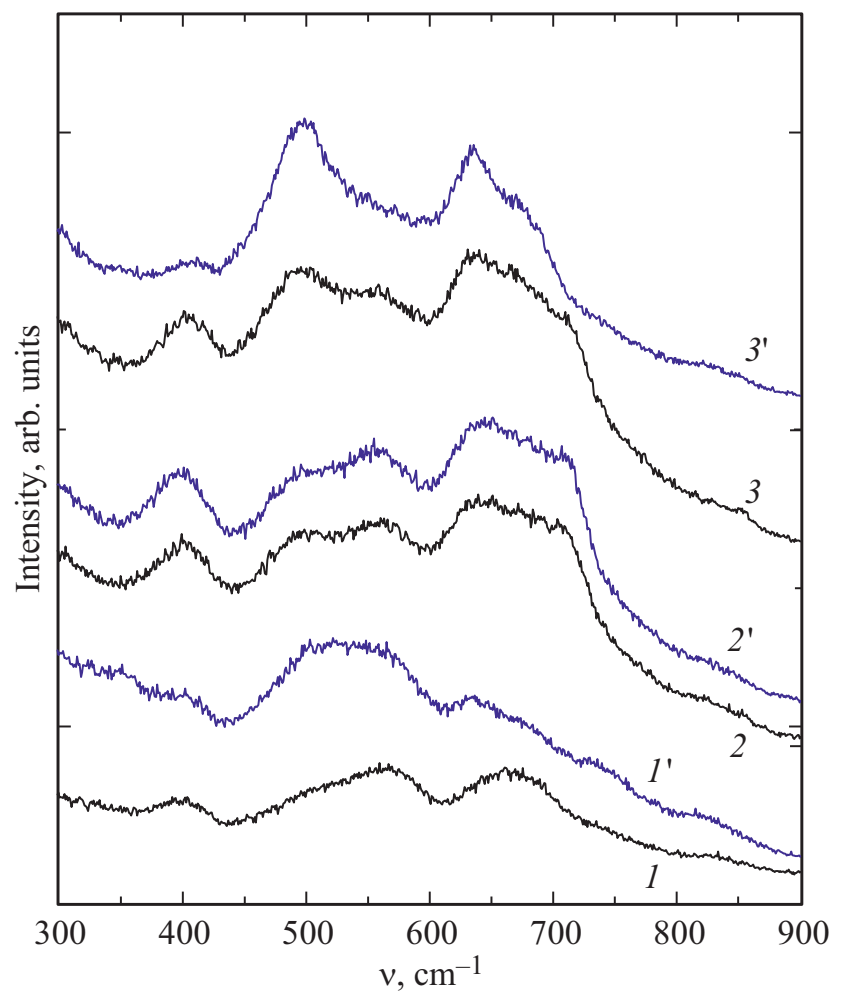

Рис. 3. КР-спектры безводных образцов $\quad \mathrm{Ba}_{4} \mathrm{In}_{2} \mathrm{Zr}_{2} \mathrm{O}_{11}(1), \quad \mathrm{Ba}_{4} \mathrm{In}_{2} \mathrm{Zr}_{2} \mathrm{O}_{10.95} \mathrm{~F}_{0.1} \quad$ (2), $\mathrm{Ba}_{4} \mathrm{In}_{2} \mathrm{Zr}_{2} \mathrm{O}_{10.95} \mathrm{Cl}_{0.1}$ (3) и гидратированных образцов $\mathrm{Ba}_{4} \mathrm{In}_{2} \mathrm{Zr}_{2} \mathrm{O}_{11} \cdot 0.95 \mathrm{H}_{2} \mathrm{O}\left(1^{\prime}\right), \mathrm{Ba}_{4} \mathrm{In}_{2} \mathrm{Zr}_{2} \mathrm{O}_{10.95} \mathrm{~F}_{0.1} \cdot 0.8 \mathrm{H}_{2} \mathrm{O}$ (2') и $\mathrm{Ba}_{4} \mathrm{In}_{2} \mathrm{Zr}_{2} \mathrm{O}_{10.95} \mathrm{Cl}_{0.1} \cdot 0.6 \mathrm{H}_{2} \mathrm{O}\left(3^{\prime}\right)$.

$\mathrm{OH}^{-}$-групп в кислород-дефицитные полиэдры и соответственно к уменьшению степени гидратации образцов в данном ряду.

Для изучения влияния процессов гидратации на локальную структуру исследуемых образцов были получены КР-спектры гидратированных составов $\mathrm{Ba}_{4} \mathrm{In}_{2} \mathrm{Zr}_{2} \mathrm{O}_{11} \cdot 0.95 \mathrm{H}_{2} \mathrm{O}, \quad \mathrm{Ba}_{4} \mathrm{In}_{2} \mathrm{Zr}_{2} \mathrm{O}_{10.95} \mathrm{~F}_{0.1} \cdot 0.8 \mathrm{H}_{2} \mathrm{O}$ и $\mathrm{Ba}_{4} \mathrm{In}_{2} \mathrm{Zr}_{2} \mathrm{O}_{10.95} \mathrm{Cl}_{0.1} \cdot 0.6 \mathrm{H}_{2} \mathrm{O}$. Сравнение спектров безводных и гидратированных образцов приведено на рис. 3 . Как видно, наиболее значимые изменения регистрируются для сигнала $\sim 660 \mathrm{~cm}^{-1}$, интенсивность которого уменьшается для гидратированных образцов относительно безводных. Аналогичные результаты были получены ранее для матричного состава $\mathrm{Ba}_{4} \mathrm{In}_{2} \mathrm{Zr}_{2} \mathrm{O}_{11}$ [24].

Установление форм кислородно-водородных групп было выполнено с помощью метода ИК спектроскопии (рис. 4). Для всех рассматриваемых фаз в спектрах регистрируется широкая полоса в области $2800-3550 \mathrm{~cm}^{-1}$, что свидетельствует о присутствии кислородно-водородных групп. Отсутствие полос в области деформационных колебаний $\sim 1600$ и $\sim 1700 \mathrm{~cm}^{-1}$ подтверждает, что в структуре сложных оксидов нет молекул воды и ионов гидроксония. В данной области фиксируются две пары близкорасположенных полос с частотами 1370 и $1420 \mathrm{~cm}^{-1}$, относящиеся к деформацион- 


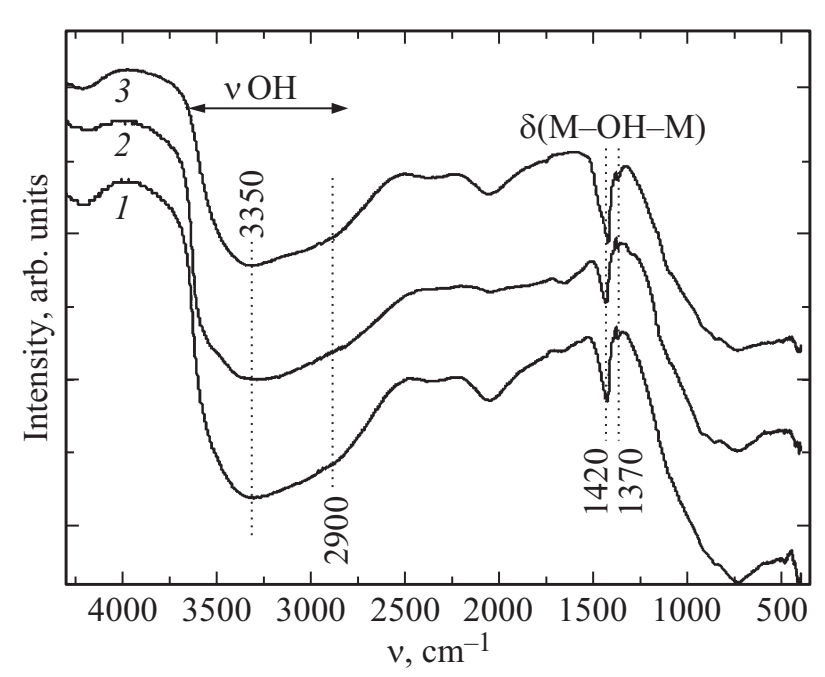

Рис. 4. ИК спектры гидратированных образцов $\mathrm{Ba}_{4} \mathrm{In}_{2} \mathrm{Zr}_{2} \mathrm{O}_{11} \cdot 0.95 \mathrm{H}_{2} \mathrm{O}$ (1), $\mathrm{Ba}_{4} \mathrm{In}_{2} \mathrm{Zr}_{2} \mathrm{O}_{10.95} \mathrm{~F}_{0.1} \cdot 0.8 \mathrm{H}_{2} \mathrm{O}$ (2), $\mathrm{Ba}_{4} \mathrm{In}_{2} \mathrm{Zr}_{2} \mathrm{O}_{10.95} \mathrm{Cl}_{0.1} \cdot 0.6 \mathrm{H}_{2} \mathrm{O}$ (3).

ным колебаниям $\delta(\mathrm{M}-\mathrm{OH}-\mathrm{M})[25,26]$, другими словами, это подтверждает наличие мостиковых $\mathrm{OH}^{-}$-групп. Поскольку единственной формой существования кислородно-водородных групп в исследуемых фазах являются $\mathrm{OH}^{-}$-группы, то сложный вид широкой полосы $2800-3500 \mathrm{~cm}^{-1} v(\mathrm{OH})$ свидетельствует о наложении нескольких сигналов, т.е. о наличии $\mathrm{OH}^{-}$-групп в различных кристаллографических положениях. Четко прослеживается наличие двух сигналов 3350 и $2900 \mathrm{~cm}^{-1}$, что позволяет говорить о двух неэквивалентных позициях $\mathrm{OH}^{-}$-групп.

Природа галогенид-ионов принципиально не влияет на вид спектров, т. е. состав кислородно-водородных групп для допированных образцов значимо не изменяется. Однако при уменьшении степени гидратации наблюдается снижение интенсивности полосы в области валентных колебаний, что подтверждает уменьшение концентрации $\mathrm{OH}^{-}$-групп в структуре. Смещения положения полос при допировании также не наблюдалось, т. е. изменений в энергетике водородных связей не происходило.

Таким образом, в работе осуществлен синтез галогензамещенных перовскитов $\mathrm{Ba}_{4} \mathrm{In}_{2} \mathrm{Zr}_{2} \mathrm{O}_{10.95} \mathrm{~F}_{0.1}$ и $\mathrm{Ba}_{4} \mathrm{In}_{2} \mathrm{Zr}_{2} \mathrm{O}_{10.95} \mathrm{Cl}_{0.1}$, рентгенографически подтверждена их однофазность. Установлено влияние природы ионазаместителя на параметры кристаллической решетки. Доказана способность исследуемых фаз к гидратации и формированию в структуре энергетически неравноценных $\mathrm{OH}^{-}$-групп. Установлено, что введение галогенид-иона приводит к уменьшению степени гидратации относительно недопированного состава $\mathrm{Ba}_{4} \mathrm{In}_{2} \mathrm{Zr}_{2} \mathrm{O}_{11}$.

Исследование выполнено за счет гранта Российского научного фонда (проект № 18-73-00006).

\section{Список литературы}

[1] Ravez J., Peraudeau J.G., Arend H., Abrahams S.C., Hagenmuller P.A. // Ferroelectrics. 1980. V. 26. P. 767.

[2] Fokina V.D., Flerov I.N., Gorev M.V., Molokeev M.S., Vasiliev A.D., Laptash N.M. // Ferroelectrics. 2007. V. 347. P. 60.

[3] Flerov I.N., Gorev M.V., Tressaud A., Grannec J. // Ferroelectrics. 1998. V. 217. P. 21.

[4] Pogoreltsev E., Flerov I., Laptash N. // Ferroelectrics. 2010. V. 401 . P. 407.

[5] Al-Mamouri M., Edwards P.P., Greaves C., Slaski M. // Nature. 1994. V. 369. P. 382.

[6] Francesconi M.G., Slater P.R., Hodges J.P., Greaves C., Edwards P.P., Al-Mamouri M., Slaski M.J. // Solid State Chem. 1998. V. 135. P. 17.

[7] Chen J.M., Nachimuthu P., Liu R.S., Lees S.T., Gibbons K.E., Gameson I., Jones M.O., Edwards P.P. // Phys. Rev. B. 1999. V. 60. P. 6888.

[8] Greaves C., Francesconi M.G. // Current Opinion in Solid State and Materials Science. 1998. V. 132. P. 132.

[9] Robbins M., Pierc R.D., Wolfe R. // J. of Physics and Chemistry of Solids. 1971. V. 32. P. 1789.

[10] Antipov E.V., Abakumov A.M. Functionalized Inorganic Fluorides. / Ed. by Alain Tressaud. Wiley-VCH, 2010.

[11] Kosova N.A., Resepova N.O. // Materials Today: Proc. 2017. V. 4. P. 4496.

[12] Kosova N.A., Resepova N.O., Petrov S.A., Slobodyuk A.B. // J. Electrochemical Society A. 2017. V. 164. P. 6192.

[13] Kosova N.A., Slobodyuk A.B., Podgornova O.A. // J. Structural Chemistry. 2016. V. 57. P. 345.

[14] Tarasova N., Animitsa I. // J. Alloys and Compounds. 2018. V. 739. P. 353.

[15] Tarasova N.A., Animitsa I.E. // Opt. and Spectrosc. 2017. V. 123. P. 44.

[16] Tarasova N.A., Animitsa I.E. // Russian J. Electrochemistry. 2017. V. 53. P. 734.

[17] Animitsa I.E., Dogodaeva E.N., Nokhrin S.S., Kosareva, O.A., Neiman, Ya A. // Russian J. Electrochemistry. 2010. V. 46. P. 734.

[18] Shannon R.D. // Acta Crystallographica A. 1976. V. 32. P. 155.

[19] Last J.T. // Phys. Rev. 1951. V. 105. P. 1740.

[20] Tarasova N.A., Animitsa I.E. // Opt. and Spectrosc. 2017. V. 123. P. 44.

[21] Rolle A., Daviero-Minaud S., Roussel P., Rubbens A., Vannier R.N. // Solid State Ionics. 2008. V. 179. P. 771.

[22] Bielecki J., Parker S.F., Ekanayake D., Rahman S.M.H., Borjesson L., Karlsson M.J. // Materials Chemistry A. 2014. V. 2. P. 16915.

[23] Shi F., Dong H., Liu Q., Yang Y., Ren S., Sun H., Xiong J. // J. Mater. Sci.: Mater Electron. 2017. V. 28. P. 3467.

[24] Karlsson M., Matic M., Knee Ch., Ahmed I., ErikssonS., Börjesson L. // Chemistry of Materials. 2008. V. 20. P. 3480.

[25] Юхневич Г.В. Инфракрасная спектроскопия воды. М.: Наука, 1973. 205 с.

[26] КарякинА.В., Кривенцова Г.А. Состояние воды в неорганических и органических соединениях. М.: Наука, 1973. $176 \mathrm{c}$. 